\title{
Dynamical star-disk interaction in the young stellar system V354 Monocerotis ${ }^{\star}$
}

\author{
N. N. J. Fonseca ${ }^{1,2,3}$, S. H. P. Alencar ${ }^{1}$, J. Bouvier ${ }^{2}$, F. Favata ${ }^{4}$, and E. Flaccomio ${ }^{5}$ \\ ${ }^{1}$ Departamento de Física - ICEx - UFMG, Av. Antônio Carlos, 6627, 31270-901, Belo Horizonte, MG, Brazil \\ e-mail: nath@fisica.ufmg.br \\ 2 UJF-Grenoble 1/CNRS-INSU, Institut de Planétologie et d'Astrophysique de Grenoble (IPAG) UMR 5274, 38041 Grenoble, France \\ 3 CAPES Foundation, Ministry of Education of Brazil, Brasília DF 70040-020, Brazil \\ ${ }^{4}$ European Space Agency, 8-10 rue Mario Nikis, 75738 Paris Cedex 15, France \\ 5 Istituto Nazionale di Astrofisica, Osservatorio Astronomico di Palermo G.S. Vaiana, Piazza del Parlamento 1, 90134 Palermo, Italy
}

Received 11 December 2013 / Accepted 9 May 2014

\begin{abstract}
Aims. The main goal of this work is to characterize the mass accretion and ejection processes of the classical T Tauri star V354 Mon, a member of the young stellar cluster NGC 2264.

Methods. In March 2008, photometric and spectroscopic observations of V354 Mon were obtained simultaneously with the CoRoT satellite, the $60 \mathrm{~cm}$ telescope at the Observatório Pico dos Dias (LNA, Brazil) equipped with a CCD camera and Johnson/Cousins $B V(R I)_{\mathrm{c}}$ filters, and the SOPHIE échelle spectrograph at the Observatoire de Haute-Provence (CNRS, France).

Results. The light curve of V354 Mon shows periodical minima $(P=5.26 \pm 0.50$ days $)$ that vary in depth and width at each rotational cycle. The $B V(R I)_{\mathrm{c}}$ observations indicate that the system becomes slightly bluer as the flux increases. The spectra of this T Tauri star exhibit variable emission lines, with blueshifted and redshifted absorption components associated with a disk wind and with the accretion process, respectively, confirming the magnetospheric accretion scenario. From the analysis of the photometric and spectroscopic data, it is possible to identify correlations between the emission line variability and the light-curve modulation of the young system, such as the occurrence of pronounced redshifted absorption in the $\mathrm{H} \alpha$ line at the epoch of minimum flux. This is evidence that during photometric minima we see the accretion funnel projected onto the stellar photosphere in our line of sight, implying that the hot spot coincides with the light-curve minima. We applied models of cold and hot spots and a model of occultation by circumstellar material to investigate the source of the observed photometric variations.

Conclusions. We conclude that nonuniformly distributed material in the inner part of the circumstellar disk is the main cause of the photometric modulation, which does not exclude the presence of hot and cold spots at the stellar surface. It is believed that the distortion in the inner part of the disk is created by the dynamical interaction between the stellar magnetosphere, inclined with respect to the rotation axis, and the circumstellar disk, as also observed in the classical T Tauri star AA Tau and predicted by magnetohydrodynamical numerical simulations.
\end{abstract}

Key words. stars: pre-main sequence - techniques: photometric - techniques: spectroscopic - accretion, accretion disks

\section{Introduction}

The study of young stellar objects is important for understanding the phenomena that occur in star and planet formation, including our solar system. T Tauri stars are young $\left(\sim 10^{6}\right.$ years $)$, low-mass stars $\left(M \leq 2 M_{\odot}\right)$ in the pre-main sequence (PMS) phase, which are of great interest as prototypes of young solar-type stars. They emit X-rays and have strong magnetic fields ( $2 \mathrm{kG}$, Johns-Krull et al. 2001). Based on their $\mathrm{H} \alpha$ emission strength, they are classified as classical (CTTSs) or weak-line T Tauri stars (WTTSs). CTTSs have a flux excess with respect to the stellar photosphere at infrared, optical, and ultraviolet wavelengths, which is not observed in WTTSs.

* Based on the observations obtained with the CoRoT satellite, at the Observatório Pico dos Dias, Brazil, and at the Observatoire de Haute Provence, France. The CoRoT space mission was developed and is operated by the French space agency CNES, with participation of ESA's RSSD and Science Programmes, Austria, Belgium, Brazil, Germany, and Spain.
The CTTSs also exhibit irregular photometric and spectroscopic variability, with broad emission lines, strong $\mathrm{H} \alpha$ emission, and forbidden emission lines. Most of these general features are reproduced by magnetospheric accretion models (Shu et al. 1994; Hartmann et al. 1994; Muzerolle et al. 2001; Kurosawa et al. 2006; Lima et al. 2010), in which a young magnetized star accretes material from a circumstellar disk. The stellar magnetic field is strong enough to disrupt the disk at a distance of few stellar radii from the star. Ionized material from the inner disk is then channeled onto the stellar surface along field lines, creating accretion funnels. Hot spots are produced at the stellar surface by the strong shock of material at free-fall velocity. Material is also ejected from the system in the form of a stellar wind and as a disk wind along open magnetic field lines. From this model, we can explain some characteristics observed in CTTSs. Hot spots are responsible for optical and ultraviolet excess emission. The broad emission lines, which usually present redshifted absorptions, are predominantly produced by accelerated material in the accretion funnels. Blueshifted absorptions and forbidden emission lines are formed in the low-density 
wind. Infrared excess emission comes from the reprocessing by the disk of radiation generated in the system.

Although the magnetospheric accretion scenario describes the general CTTSs properties, some observational results indicate that this axisymmetric, stable model is not completely correct (Bouvier et al. 2007b). Some studies demonstrated that the outflow and inflow processes are intimately connected (Cabrit et al. 1990; Hartigan et al. 1995). The interaction between the stellar magnetosphere and the disk is expected to be very dynamic, as shown by magnetohydrodynamical (MHD) simulations (Goodson \& Winglee 1999; Romanova et al. 2002). As a result of differential rotation between the star and the inner disk region where the accretion flux originates, the field lines may be distorted after a few rotational periods, eventually reconnecting and restoring the initial field configuration. This process repeats as the star rotates. Furthermore, a misalignment between the rotation and magnetic axes creates a deformation in the inner disk, leading to the formation of non-axisymmetric regions where accretion is favored (Terquem \& Papaloizou 2000; Romanova et al. 2003).

One of the best-studied CTTSs is AA Tau, observed for a month during three different campaigns (Bouvier et al. 1999, 2003, 2007a). This star exhibits a peculiar photometric behavior, with almost constant brightness interrupted by quasi-cyclical and irregular episodes of attenuation. The brightness decrease occurs in absence of significant color variation and with an increase of polarization (Ménard et al. 2003). This was interpreted as occultation of the stellar photosphere by circumstellar dusty material present in an inner disk warp. The warp is produced by the interaction between the disk and the stellar magnetic field, misaligned with respect to the rotation axis, as suggested by MHD simulations. Simultaneous high-resolution spectroscopy has shown signs of correlation between mass accretion and ejection processes. During the second observing campaign, the photometric and spectroscopic variability was drastically reduced for a few days, revealing an episode of disruption of magnetic configuration at the inner edge of the disk, which suppressed accretion temporarily. The observed variations in $\mathrm{H} \alpha$ absorption components showed the cyclical process of inflation and reconnection of field lines caused by the differential rotation between the star and the inner disk region, once again in agreement with MHD simulations. The same evidence of this dynamical interaction was observed again in the third campaign, five years after the second one.

Even though the photometric behavior of AA Tau was atypical at that time, the characteristics of the structure of its accretion zone might be similar in other CTTSs. To confirm this hypothesis, it would be necessary to obtain high-precision photometry for many CTTSs during many rotational periods. This corresponds to a continuous observation of at least a month, based on typical rotational periods of eight days for CTTSs in Taurus. Such a monitoring from the ground is complicated because of telescope-time allocation and weather conditions. The CoRoT satellite additional program to observe the star formation region NGC 2264 during 23 days uninterruptedly in 2008 allowed this analysis to be performed.

NGC 2264 is a well-studied young stellar cluster, located in the Mon OB1 association at $\sim 760$ pc (Sung et al. 1997; Gillen et al. 2014), with evidence of active star formation (Dahm 2008). The pioneering work of Herbig (1954), Walker (1956), and others established this region as an important laboratory for studies of star formation and evolution of young stars. From the light curves of 83 CTTSs identified among the 301 cluster members observed with CoRoT in 2008, 23 have been classified as
Table 1. Journal of observations.

\begin{tabular}{lccc}
\hline \hline Dates (March 2008) & Instrument & Exp. time (s) & $N_{\text {obs }}$ \\
\hline Spectroscopy & & & \\
$12,14-1,14-2$, & SOPHIE & 3600 & 10 \\
$18-1,18-2,20$, & (OHP) & & \\
$23,25,27$, and 28 & & & \\
\hline Photometry & & & \\
7 to 30 & CoRoT & 512 & cont. \\
$19,20,25,26$, and 27 & $60 \mathrm{~cm}$ & $B 400$ & 10 \\
& (OPD) & $V 300$ & 8 \\
& & $R 200$ & 8 \\
& & $I 200$ & 10 \\
\hline
\end{tabular}

Notes. On March 14 and 18, we obtained two spectra of V354 Mon, referred to as 14-1, 14-2 and 18-1, 18-2.

exhibiting the same type of variability as AA Tau (Alencar et al. 2010). This revealed that the photometric behavior of AA Tau is common in young stellar objects; it was found in $28 \% \pm 6 \%$ of the CTTSs in NGC 2264 observed with CoRoT. These systems are essential to test predictions of MHD simulations.

The main goal of the work presented in this paper is to characterize the mass accretion and ejection properties of the CTTS V354 Mon, a member of NGC 2264, from simultaneous high-resolution photometric and spectroscopic observations. V354 Mon is one of the 23 cluster members whose light curves have been classified as AA Tau type. From the analysis of these data, we identify correlations between emission line variability and light-curve modulation. It is possible to investigate the dynamical processes of mass accretion and outflow that occur in this system, as well as the interaction between the stellar magnetic field and the circumstellar disk. We also test predictions of magnetospheric accretion models and MHD simulations, constructing plausible scenarios for the phenomena observed in this young object.

The paper is organized as follows: Sect. 2 describes the observations and data reduction. In Sect. 3 we present the data analysis and results. In Sect. 4 we discuss the possible causes of the observed photometric and spectroscopic variations. The conclusions are depicted in Sect. 5.

\section{Observations}

CoRoT observed NGC 2264 from March 7 to 30, 2008. For V354 Mon, a cluster member in the CoRoT observational program, high-resolution spectroscopy and $B V(R I)_{\mathrm{c}}$ photometry were obtained simultaneously with the SOPHIE échelle spectrograph at the Observatoire de Haute-Provence (OHP, CNRS, France) and the $60 \mathrm{~cm}$ telescope equipped with a CCD camera and Johnson/Cousins filters at the Observatório Pico dos Dias (OPD, LNA, Brazil), respectively. The journal of observations is given in Table 1. V354 Mon is a CTTS with a well-determined photometric period, which increases the chances that this system is being seen edge-on, and exhibits a large $\mathrm{H} \alpha$ equivalent width, which facilitates the study of its spectroscopic variability with good signal-to-noise ratio, at least in this line. Information from the literature about V354 Mon is gathered in Table 2.

Many results presented in this work are dependent on the effective temperature of the star. Lamm et al. (2004) assigned a K4 spectral type to V354 Mon, which agrees with the $T_{\text {eff }}$ value of $4590 \mathrm{~K}$ obtained by Flaccomio et al. (2006), using the Kenyon \& Hartmann (1995) spectral type/intrinsic color scale that is, however, more appropriate for main-sequence dwarfs than young stars. V354 Mon was observed in 2011 with the 
Table 2. Characteristics of V354 Mon from the literature.

\begin{tabular}{lc}
\hline \hline Data & Ref. \\
\hline$V=14.45$ mag & 1 \\
Spectral type: K4V & 2 \\
Photometric period $=5.22 \pm 0.87$ days & 2 \\
$\mathrm{H} \alpha$ equivalent width $=16.60 \AA$ & 1 \\
$\mathrm{H} \alpha$ width at $10 \%>270 \mathrm{~km} \mathrm{~s}^{-1}$ & 3 \\
$\log \left(T_{\text {eff }} / \mathrm{K}\right)=3.66$ & 4 \\
$\log \left(L_{\mathrm{bol}} / L_{\odot}\right)=0.34$ & 4 \\
Mass $=1.50 M_{\odot}$ & 4 \\
$\log ($ age $/$ years $)=6.42$ & 4 \\
Heliocentric radial velocity (Dec. 2004$): 19.42 \mathrm{~km} \mathrm{~s}^{-1}$ & 3 \\
\hline
\end{tabular}

References. (1) Dahm \& Simon (2005); (2) Lamm et al. (2004); (3) Fúrész et al. (2006); (4) Flaccomio et al. (2006).

FLAMES spectrograph (VLT/ESO) during a multiwavelength campaign of the NGC 2264 star-forming region (Cody et al. 2014) when 22 medium-resolution spectra $(R=17000)$ were acquired. To check the V354 Mon spectral type, we estimated the effective temperature of V354 Mon with the FLAMES spectra, using line ratios. A series of synthetic spectra were calculated with the code Spectroscopy Made Easy (SME) (Valenti \& Piskunov 1996), using the same resolution as the FLAMES observations, within the spectral domain of the FLAMES data (6440 $\mathrm{A}<\lambda<6820 \AA$ ) and the $3500 \mathrm{~K}$ to $6000 \mathrm{~K}$ temperature range, with $\log g=4.0$ and 4.5 and solar metallicity. In this range of $T_{\text {eff }}$ and $\log g, \mathrm{FeI}$ lines are very $T_{\text {eff }}$ dependent, while CaI lines are not. The $\mathrm{CaI} / \mathrm{FeI}$ ratio is therefore a $\operatorname{good} T_{\text {eff }}$ indicator that is also independent of veiling. We analyzed the CaI $6717.7 \AA$ and FeI $6546.2 \AA$ lines that were always present and easily identified in the spectra. Comparing the line ratios measured in the observed and theoretical spectra, we obtained for V354 Mon $T_{\text {eff }}=4647 \pm 161 \mathrm{~K}$ with $\log g=4.5$ (dwarfs) and $T_{\text {eff }}=4434 \pm 133 \mathrm{~K}$ with $\log g=4.0$ (young stars). The errors come from the standard deviation of the values obtained with all the FLAMES spectra. Recently, Pecaut \& Mamajek (2013) compiled tables of effective temperature vs. spectral type for both dwarfs and young (5-30 Myr) stars. We compared the $T_{\text {eff }}$ values we obtained for V354 Mon with their respective tables, and they are both consistent with a $\mathrm{K} 4$ spectral type. Recently, Mariñas et al. (2013) assigned a K7 spectral type to V354 Mon (their target 26) based on an $[\mathrm{OH}] /[\mathrm{MgI}]$ line ratio calibration obtained from $H$ band low-resolution spectra. They acknowledged an error of two subclasses to spectral types obtained with their calibration. However, in the K7-K3 spectral range, the $\mathrm{OH}(1.69 \mu \mathrm{m})$ line is about 5 to 10 times shallower than the MgI $(1.50 \mu \mathrm{m})$ line, which can complicate its evaluation in low-resolution spectra. This, together with the scatter present in their calibration, possibly adds some more uncertainty to their spectral type determination in the K7-K3 region. Our results based on the FLAMES spectra are not compatible with a K7 spectral type, which would require much lower $T_{\text {eff }}$ values $(4050 \mathrm{~K}$ for dwarfs and $3970 \mathrm{~K}$ for young stars, according to the tables of Pecaut \& Mamajek 2013) than we obtained. We decided therefore to adopt a K4 spectral type for V354 Mon, in agreement with the results of Lamm et al. (2004) and Flaccomio et al. (2006).

\subsection{Photometry}

The CoRoT data were reduced using a standard procedure (Samadi et al. 2007; Auvergne et al. 2009) and delivered in the form of a light curve. The light curve was processed using a sigma-clipping filter that removed outliers (mostly related to South Atlantic Anomaly crossings) and hot pixels, and corrected for the effects associated with entrance into and exit from Earth eclipses. The light curve was also rebinned to $512 \mathrm{~s}$ and corresponds to the integrated flux in the CoRoT mask.

The OPD photometric observations were carried out on five nights between March 19 and 27, 2008. The $60 \mathrm{~cm}$ telescope was equipped with the CCD camera \#106 (SITe SI003AB) of $1024 \times 1024$ pixels and Johnson/Cousins $B V(R I)_{\mathrm{c}}$ filters. Integration times ranged from $200 \mathrm{~s}$ to $400 \mathrm{~s}$, depending on the filter. We processed raw images with the usual techniques within the IRAF environment, which included bias subtraction and flatfield calibration. Differential photometry between V354 Mon and a reference star was also performed using IRAF. The reference star is TYC 750-1637-1 (spectral type F7V, $V=11.609$; Sung et al. 1997), located less than 2.2' away from V354 Mon and recorded on the same images.

\subsection{Spectroscopy}

The spectroscopic observations were collected on eight nights between March 12 and 28, 2008. We obtained ten highresolution spectra at the $1.93 \mathrm{~m}$ OHP telescope with the SOPHIE dual fiber échelle spectrograph (Perruchot et al. 2008), which yields 41 orders covering the $3872 \AA$ to $6943 \AA$ domain at a mean spectral resolution of $\lambda / \Delta \lambda \simeq 75000$ (high-resolution mode), and simultaneously records the object and the neighboring sky. All spectra have the same exposure time of $3600 \mathrm{~s}$. The data were automatically reduced via a standard procedure, adapted from the software used with the HARPS spectrograph designed at the Geneva Observatory. The reduction procedure includes bias subtraction, optimum extraction of the orders, removal of cosmic rays, flat-fielding achieved through a tungsten lamp exposure, wavelength calibration with a thorium lamp exposure, cross-correlation with an appropriate numerical mask, and reconnection of spectral orders with barycentric correction.

Unfortunately, the nebular emission present in the stellar spectrum and in the sky spectrum are not equivalent, because the fibers used in the observations do not respond equally to the same light source ${ }^{1}$. In addition, nebular emission is highly variable on scales of arcseconds or less, according to studies of HII regions (Henney \& O’Dell 1999; McCollum et al. 2004). For these reasons, contamination by nebular emission was hard to exclude properly and some emission lines could not be analyzed. For each spectrum, we subtracted the sky continuum level, normalized the stellar continuum to unity, and corrected from the star radial velocity. Then we subtracted a template spectrum (HD 190007) of same spectral type as V354 Mon, resulting in circumstellar spectral profiles. We also calculated the average profile of the spectra obtained on the same night, since there is no significant variation between them, resulting in one spectrum for each night of observation.

\section{Results}

\subsection{Photometry}

The broadband, white-light curve of V354 Mon obtained with CoRoT (Fig. 1, top panel) displays a typical CTTS behavior,

\footnotetext{
This problem was first investigated by selecting only nebular emission lines and calculating the ratio between the highest intensity of these lines in the sky and stellar spectra. It was later confirmed by OHP technicians through private communication.
} 

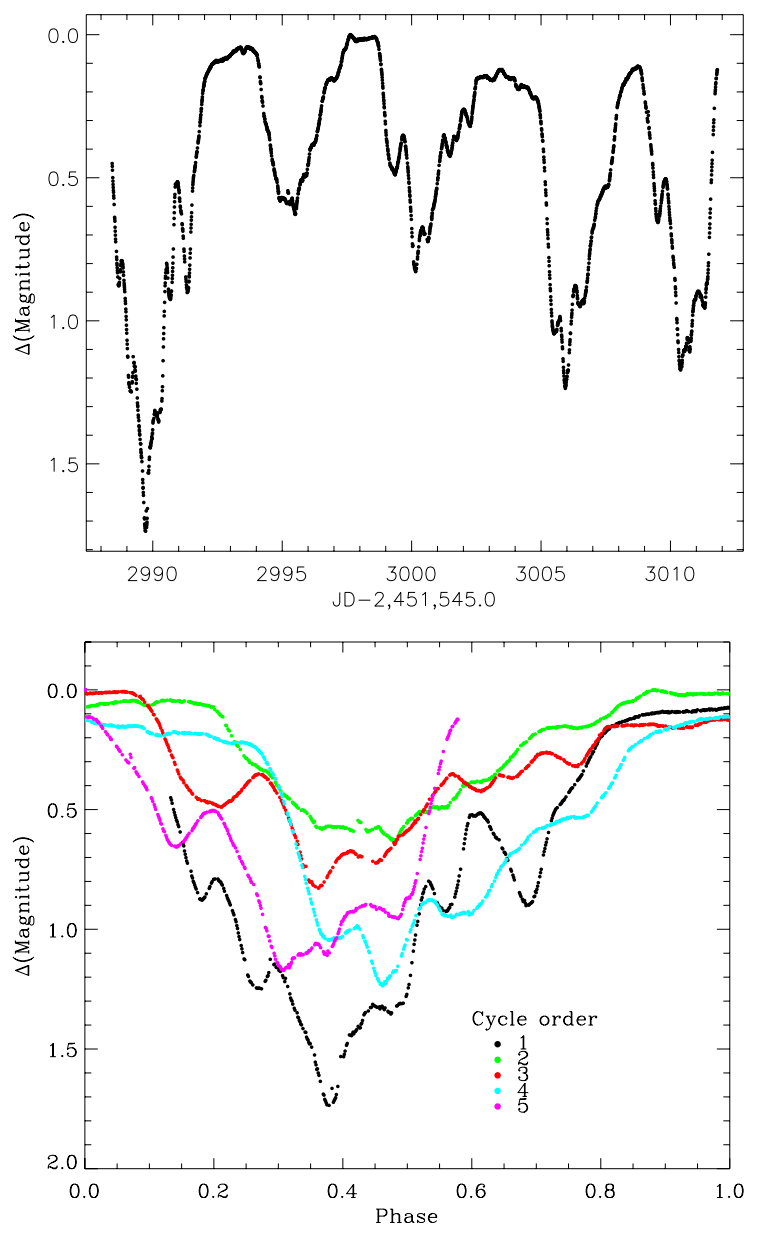

Fig. 1. CoRoT light curve, continuous (top) and folded in phase (bottom) with a period of $5.26 \pm 0.50$ days. Different colors represent different rotational cycles in the order indicated in the lower right corner of the bottom panel. Magnitudes are given on an arbitrary scale.

noticeably periodic and with well-defined minima and maxima that vary in depth and width from one rotational cycle to the other. A periodogram analysis (Scargle 1982) of the light curve reveals a period of $5.26 \pm 0.50$ days, which is consistent with the value obtained by Lamm et al. (2004), $5.22 \pm 0.87$ days. This indicates that the dominant source of photometric variability did not significantly change on a timescale of a few years. The varying depth and width at minimum is more evident when the light curve is folded in phase with the calculated period (Fig. 1, bottom panel).

We measured the percentage variability amplitude of the light curve as $\left[\left(\right.\right.$ Flux $_{\max }-$ Flux $\left._{\min }\right) /$ Flux $\left._{\text {median }}\right] \times 100$, obtaining a value of $115 \%$ for V354 Mon. This is one of the largest photometric variations in the CTTSs observed with CoRoT, which range between $3 \%$ and $137 \%$ (Alencar et al. 2010).

As observed in Fig. 2, the OPD data are consistent with the CoRoT photometry. From the calculation of magnitude differences between the observations of March 25 and 27, we note that the photometric amplitude decreases toward longer wavelengths: $1.5 \pm 0.3$ in $B, 1.15 \pm 0.05$ in $V, 0.6 \pm 0.5$ in $R$, and $0.4 \pm 0.1$ in $I$. Analyzing the color variation (Fig. 2), we see that the system becomes slightly bluer as the flux increases.

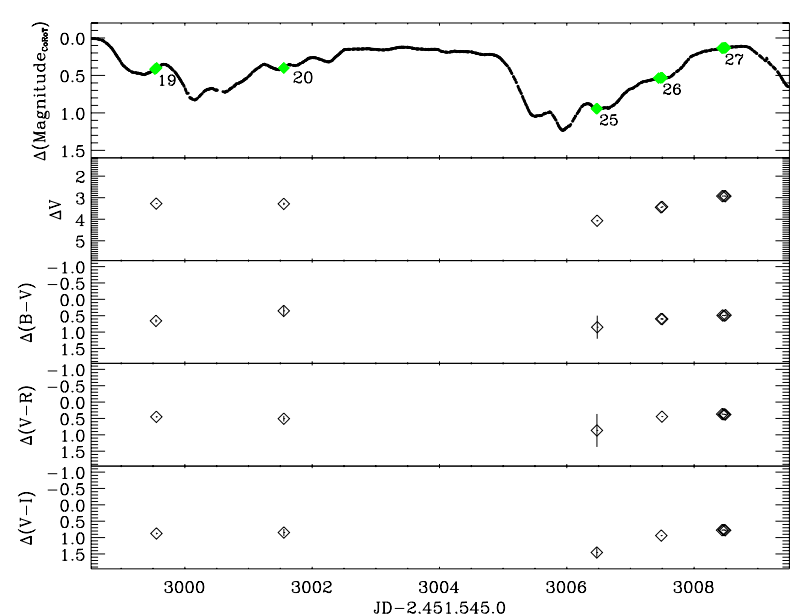

Fig. 2. CoRoT light curve, marked with OPD photometric observation dates, compared with the $V$-band photometry and color variations of V354 Mon. Vertical bars indicate the errors of each measurement. The magnitude scale on the vertical axis is the same for each color plot.

\subsection{Spectroscopy}

For the reasons stated in Sect. 2.2, contamination by nebular emission present in the stellar spectrum was hard to exclude properly, which restricted the analysis of emission lines. In this work we focus on the $\mathrm{H} \alpha$ region. To exclude the nebular emission from calculations or identify its contribution in the results, we defined the range of the contaminated spectral region. In each sky spectrum we determined the wavelength values that constrain the region around $6562.85 \AA$ where the flux is higher than the normalized background continuum. The mean values obtained are 6561.4 and $6563.4 \AA$, corresponding to velocity values of -64.6 and $27.1 \mathrm{~km} \mathrm{~s}^{-1}$, respectively, relative to the spectral line center at the stellar rest frame.

The circumstellar profiles exhibit a remarkable variability (Fig. 3), with a well-distinguishable nebular emission in the central part of the plots. We show in Fig. 4 the $\mathrm{H} \alpha$ line average profile with its normalized variance (Johns \& Basri 1995), which measures how much each region of the observed profiles varies with respect to the average profile. We note that the blueshifted region is more variable than the redshifted one. The blueshifted absorption seen in the average profile is generally associated with winds in the system.

Using the same method as was applied to calculate the lightcurve period discussed in Sect. 3.1, we investigated the $\mathrm{H} \alpha$ normalized flux periodicity through a periodogram analysis of the observed time series, which we did independently in each velocity bin of $0.5 \mathrm{~km} \mathrm{~s}^{-1}$ along the profile. The results were disposed side by side in space velocity to form an image. The twodimensional periodogram is shown in Fig. 5, where the normalized power scales from zero (white) to the highest value (black). The $\mathrm{H} \alpha$ redshifted side displays periodicity in a broad region centered on 5.3 days, which is close to the photometric period, while the blueshifted side is variable at around 3.1 days.

The period obtained for the variation of the $\mathrm{H} \alpha$ redshifted side is close to the photometric period, which is interesting from the point of view of magnetospheric accretion with a misalignment between the rotation and magnetic axes. In this scenario, material from the inner part of the disk is more easily channeled along the field lines that are deflected from the disk mid-plane towards the stellar surface because of the tilt of the stellar magnetosphere. Thus there is a preferential region of accretion, causing 


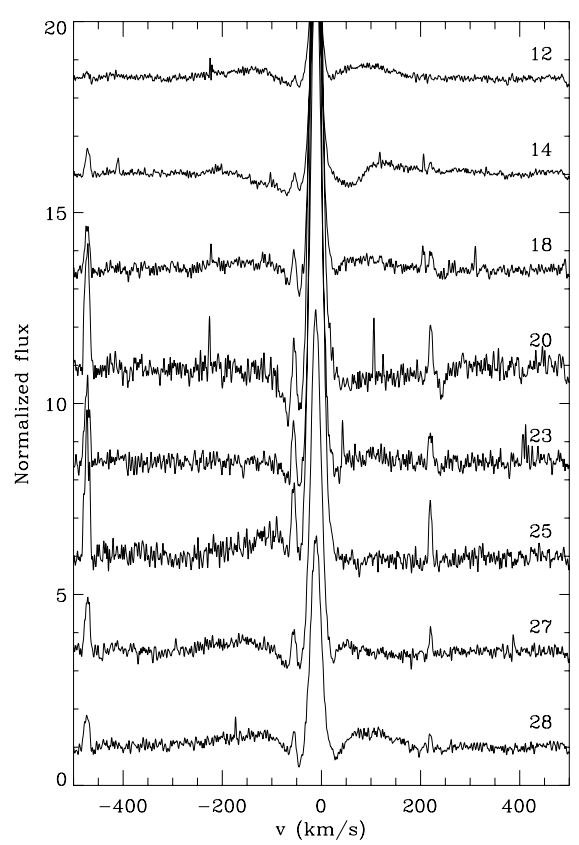

Fig. 3. $\mathrm{H} \alpha$ circumstellar spectra for each observed night, indicated at the right of each profile. The continuum of each spectrum has been normalized to unity and the profiles have been shifted for clarity.

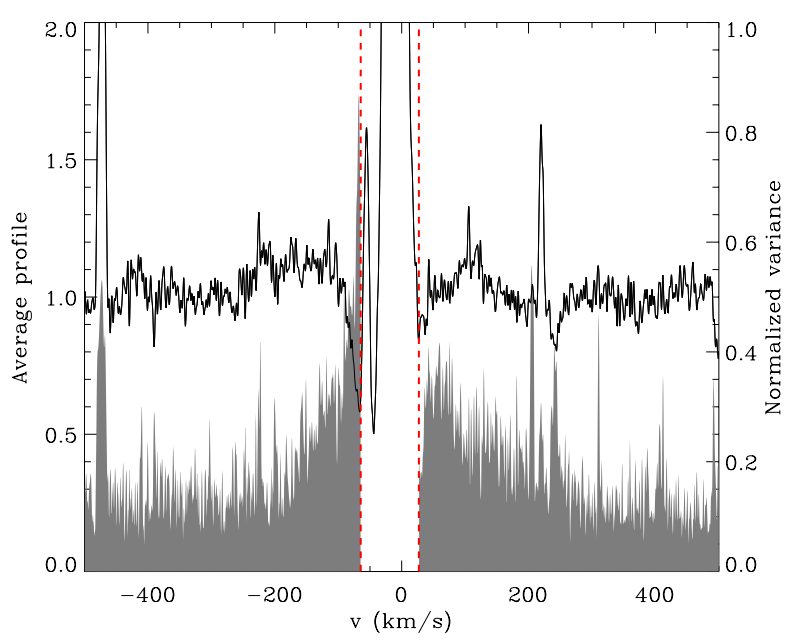

Fig. 4. Average profile (solid line) and normalized variance (gray shaded area) of $\mathrm{H} \alpha$ line. Dashed red lines delimit the region dominated by nebular emission.

the $\mathrm{H} \alpha$ redshifted absorption to vary with the stellar rotational period. This absorption will be more pronounced and at higher velocities when we see the accretion region projected onto the stellar photosphere along our line of sight.

The modulation of the $\mathrm{H} \alpha$ blueshifted side presents a period shorter than the photometric period. In this case, the existence of only one preferential region of ejection of material from the disk is not physically acceptable, as the wind generation is located immediately beyond the accretion region, at a slightly larger distance from the star, which corresponds to a Keplerian period also slightly longer. The period of $\sim 3$ days may indicate that in fact we observe two major contributions of the wind, at opposite sides. Consequently, the formation region of these components is related to a Keplerian period of $\sim 6$ days, slightly longer than the

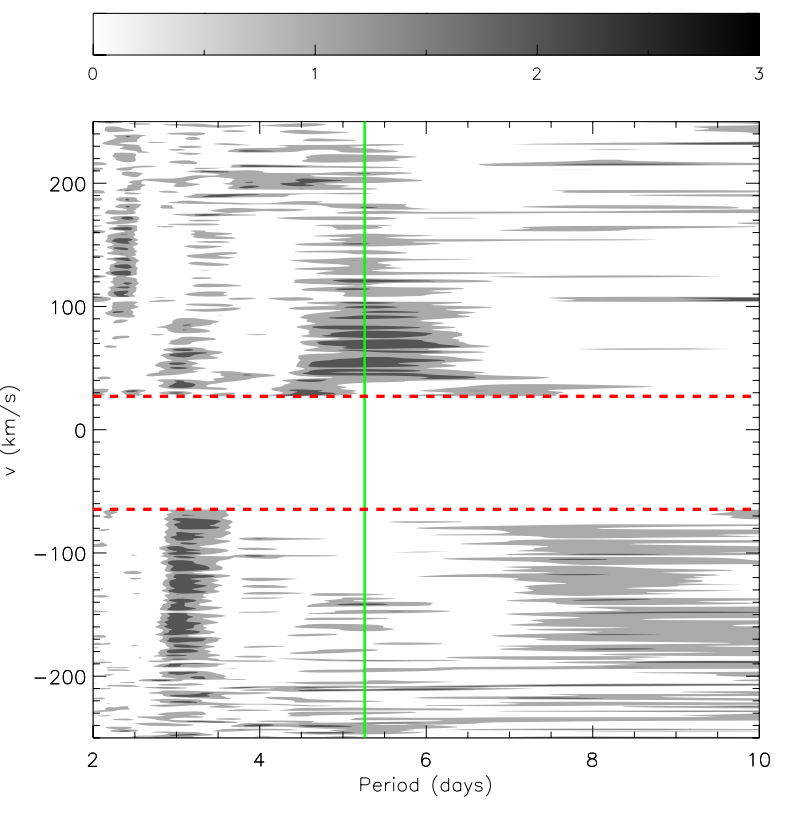

Fig. 5. Two-dimensional periodogram of $\mathrm{H} \alpha$ line flux. The power scale varies from zero (white) to the highest value (black). Dashed red lines delimit the region dominated by nebular emission. The solid green line marks the period of 5.26 days.

period of the variation of $\mathrm{H} \alpha$ redshifted side, which is connected with the accretion process.

From analyzing the $\mathrm{H} \alpha$ profiles, we observe that the spectrum obtained on March 25 shows a strong emission in the blueshifted side and no emission in the redshifted side. Because no other profile resembles it, this blueshifted emission might be a sign of a larger, occasional ejection of material, maybe related to the disruption of the magnetic field configuration. Since this isolated event is not linked to the rotational modulation of the system, we made a new analysis of the $\mathrm{H} \alpha$ flux periodicity excluding the spectrum of March 25. We recovered the results obtained considering all spectra, but the blueshifted side also presented a broad region around the photometric period, while a periodicity of about 3 days in the redshifted side becomes evident, making the new 2D periodogram symmetric. Then all $\mathrm{H} \alpha$ components shows periodic variation according to the photometric modulation. However, the period of 3 days has to be confirmed with a richer dataset because we have only a few points to investigate additional periods.

The $\mathrm{H} \alpha$ profiles are shown as a function of rotational phase with photometric period (Fig. 6) to identify similarities and differences between the spectra that are close in phase position. The top plot helps to locate the spectroscopic observations in the light curve and to identify a correlation between them. The profile of March 28 resembles the profile of March 18, with a slight difference in the redshifted absorption component. They are close to each other in phase, 0.11 and 0.20 , and at similar points of the light-curve profile, in which there is a flux decrease before a small local peak. Similarly, the spectra of days 12, 28, 23, and 18 are similar, with an asymmetric profile and a more intense redshifted side. All of them are located in the first half of the photometric cycle, in which the stellar brightness is reduced. On the other hand, the spectra of days 25, 20, and 27 are also similar, with an asymmetric profile as well, but are more intense in the blueshifted side. These observations are located in the flux increase of the light curve. The spectrum of March 14 seems to be a transition between these two situations, because it is situated 

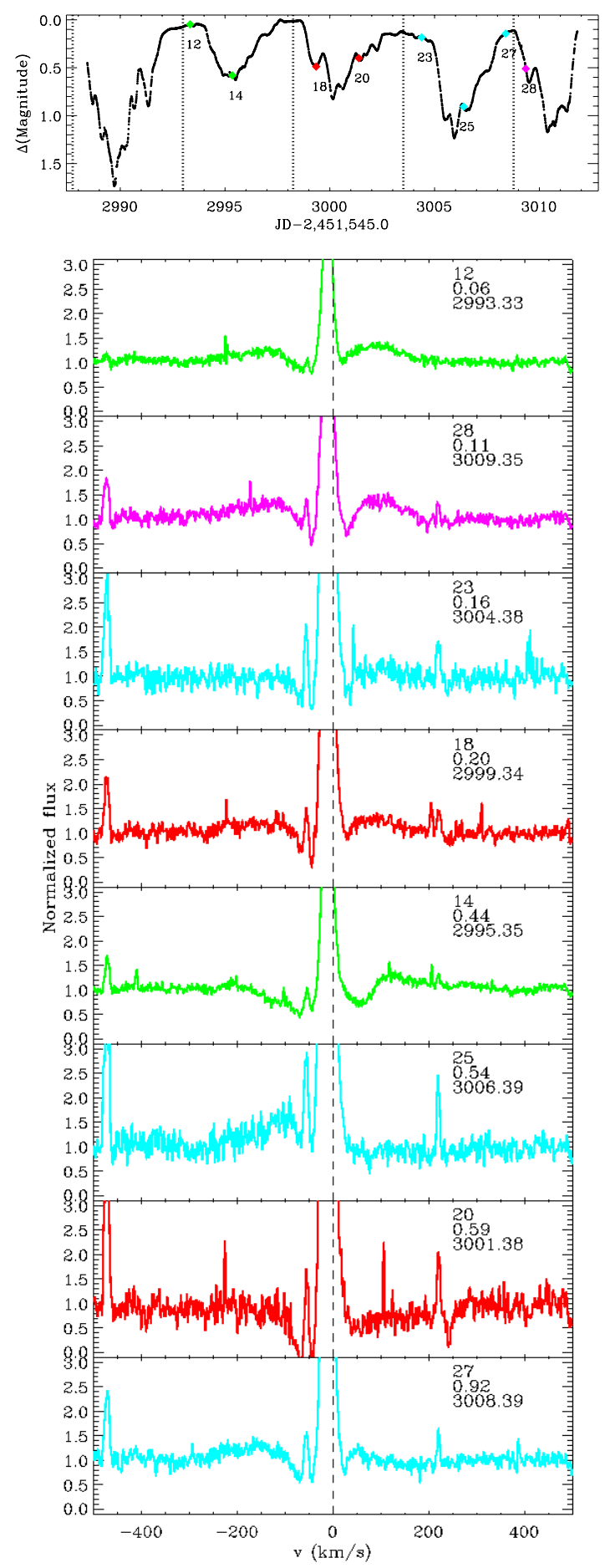

Fig. 6. $\mathrm{H} \alpha$ profiles ordered according to rotational phase (middle number in each panel). The observation date and CoRoT JD are also displayed (top and bottom numbers). The color code is the same as in Fig. 1, bottom panel. The dashed vertical line marks the central position of the line, corresponding to $6562.85 \AA$. The top panel helps to locate the spectroscopic observations in the light curve and to identify the correlation between them.

in a photometric minimum and presents a distinct profile. We conclude that there is a correlation between the light-curve modulation and the spectral line variability. The phenomenon that produces these variations appears to be asymmetric, since the brightness increase in the light curve seems to be slower than the decrease and the emission profiles observed in these two phase ranges exhibit different characteristics.

\section{Discussion}

The most often discussed causes of photometric variations at optical wavelengths in young stars are cold spots produced by magnetic activity, hot spots created by the shock of the accretion flow at the stellar photosphere, and partial occultation of the star by inhomogeneous circumstellar material (Herbst et al. 1994). Of these, only cold spots are not associated with an accretion disk. We analyze below the photometric and spectroscopic variability expected in each one of these scenarios and compare this with the observed characteristics of V354 Mon in an attempt to identify the physical phenomenon that dominates the observed optical variability in this system.

\subsection{Cold and hot spots}

Cold spots are one of the most common sources of photometric variability in low-mass stars at all ages. They are good indicators of magnetic activity, since they are associated with the eruption of magnetic flux from the stellar interior out into the atmosphere. Hot spots in young stars are caused by infalling gas at the stellar photosphere, which is a direct consequence of accretion.

Spots rotate with the star, as they are located in the photosphere, and generate periodic photometric variability on timescales of the stellar rotational period. If a cold spot is the main cause of light-curve modulation, it is fully visible to the observer at light-curve minimum, while a hot spot is fully visible at maximum. Both spots produce a modulation in the photometric amplitude that increases toward shorter wavelenghts because of the temperature difference between the spot and the photosphere, which makes the star bluer as it becomes brighter. But this effect is more pronounced for hot than for cold spots. In this manner, we can estimate the spot temperature and size by comparing the total photometric amplitude of variation at different wavelengths.

We applied the model developed by Bouvier et al. (1993), which derives the temperature and the smallest size of a spot that is responsible for the modulation of the stellar brightness through the fit of amplitude variability as a function of wavelength. This model searches for the best spot configuration that reproduces the observed amplitudes using a $\chi^{2}$ method, taking into account limb-darkening effects and simultaneously fitting the amplitudes in all bands. No assumption is made about the spot number and shape, but it is assumed that all have the same temperature and the temperature distribution in each individual spot is uniform. Therefore this model does not determine the spot location over the star and provides only a lower limit for the fractional area of the visible stellar hemisphere covered by spots.

We considered that the star has an effective temperature of $4500 \mathrm{~K}$ (Flaccomio et al. 2006), with limb-darkening coefficients consistent with this temperature given by Claret (2000) for $\log g \sim 4.0$ and $\log [\mathrm{M} / \mathrm{H}] \sim 0$. Evaluating spots with temperatures of $3.0 \leq \log \left(T_{\mathrm{s}} / \mathrm{K}\right) \leq 4.0$ and fractional area $f$ of the visible stellar hemisphere between $0.1 \%$ and $90 \%$, the model converged to a spot of $T_{\mathrm{s}}=10000 \mathrm{~K}$ and $f=5 \%$ as the best configuration that reproduces the observed amplitudes of V354 Mon. Even though this solution is at the boundary of model parameters because it presents the highest possible spot temperature, this result is plausible because it agrees with hot spot parameters derived for other $\mathrm{T}$ Tauri stars (Bouvier et al. 1995). 
Although the spot model indicates that hot spots can reproduce the amplitudes of variation better than cold spots, we restricted the fit to spots with $T_{\mathrm{s}}<4500 \mathrm{~K}$ to confirm that cold spots cannot be considered as the main cause of photometric modulation. In this case, we obtained $T_{s}=3981 \mathrm{~K}$ and $f=89 \%$ as the best configuration. But this result is implausible because cold spots in TTSs typically cover less than $50 \%$ of the projected stellar disk (Bouvier et al. 1995). We conclude that cold spots probably exist on the stellar surface of V354 Mon, but are not the main cause of the light-curve variability.

The effective temperature of V354 Mon adopted from Flaccomio et al. (2006) was derived based on the Kenyon \& Hartmann (1995) spectral type/intrinsic color scale, which is more adequate to main-sequence dwarfs than young stars. As discussed in Sect. 2, Pecaut \& Mamajek (2013) have recently compiled a table of effective temperature vs. spectral type for 5-30 Myr old PMS stars. According to this scale, a K4 star presents an effective temperature of $4330 \mathrm{~K}$. Considering this value, the spot model yields results that are very similar to those obtained for $4500 \mathrm{~K}: T_{\mathrm{s}}=10000 \mathrm{~K}$ and $f=4 \%$ as the best configuration that reproduces the observed amplitudes of V354 Mon, and $T_{\mathrm{s}}=3852 \mathrm{~K}$ and $f=89 \%$ if we restrict the model to cold spots only.

A hot spot influences not only the light curve of the star, but also its spectrum because the accretion shock produces a continuum flux excess with respect to the stellar photosphere that veils the observed photospheric lines, reducing their depth. During the process of data reduction, we found no evidence of veiling, as shown in Fig. 7. We calculated the flux generated by a hot spot of $10000 \mathrm{~K}$ and $f=5 \%$, the best spot configuration that reproduces the observed amplitudes of V354 Mon in the $B V(R I)_{\mathrm{c}}$ bands, through a blackbody curve integration for $10000 \mathrm{~K}$ convolved with passband response functions (Bessell 1983). The ratio between the values obtained and the stellar blackbody flux without a spot at $4500 \mathrm{~K}$ was 2.84 in $B, 1.34$ in $V$, 0.86 in $R$, and 0.55 in $I$. This means that this hot spot should produce a remarkable veiling that would be measurable when comparing absorption lines observed at photometric maximum and minimum. We note in the top panel of Fig. 8 that the LiI line, one of the best-defined absorptions in the spectrum, displays no difference in depth between these two occasions. To exemplify that the flux of a spot of $10000 \mathrm{~K}$ and $f=5 \%$ would cause a visible effect, we added the calculated veiling to the LiI absorption observed at photometric maximum, when the hot spot would be fully visible. We applied the values obtained for $R$ and $I$ bands, as they have characteristic wavelengths of $5925 \AA$ and $7900 \AA$, and the LiI line is located at $6707.8 \AA$. The result is shown in the bottom panel of Fig. 8, in which we observe that there is a noticeable decrease in the line depth due to the added veiling. The fact that we do not observe this in the spectra of V354 Mon refutes a hot spot as the main cause of photometric variability.

Furthermore, if a hot spot were responsible for the observed photometric variations, the light-curve maximum would correspond to the moment when the hot spot is fully visible to the observer and the minimum would correspond to the opposite situation, when it is completely hidden. The material in free fall in the accretion funnel absorbs photons emitted by the hot spot. Thus, the $\mathrm{H} \alpha$ redshifted absorption should occur at light-curve maximum, since the spot would be in our line of sight at this moment. The opposite is observed in V354 Mon spectra, however. The $\mathrm{H} \alpha$ redshifted absorption is visible in the March 14 spectrum, located at a light-curve minimum, but is not present in the March 12 spectrum, corresponding to a light-curve maximum,
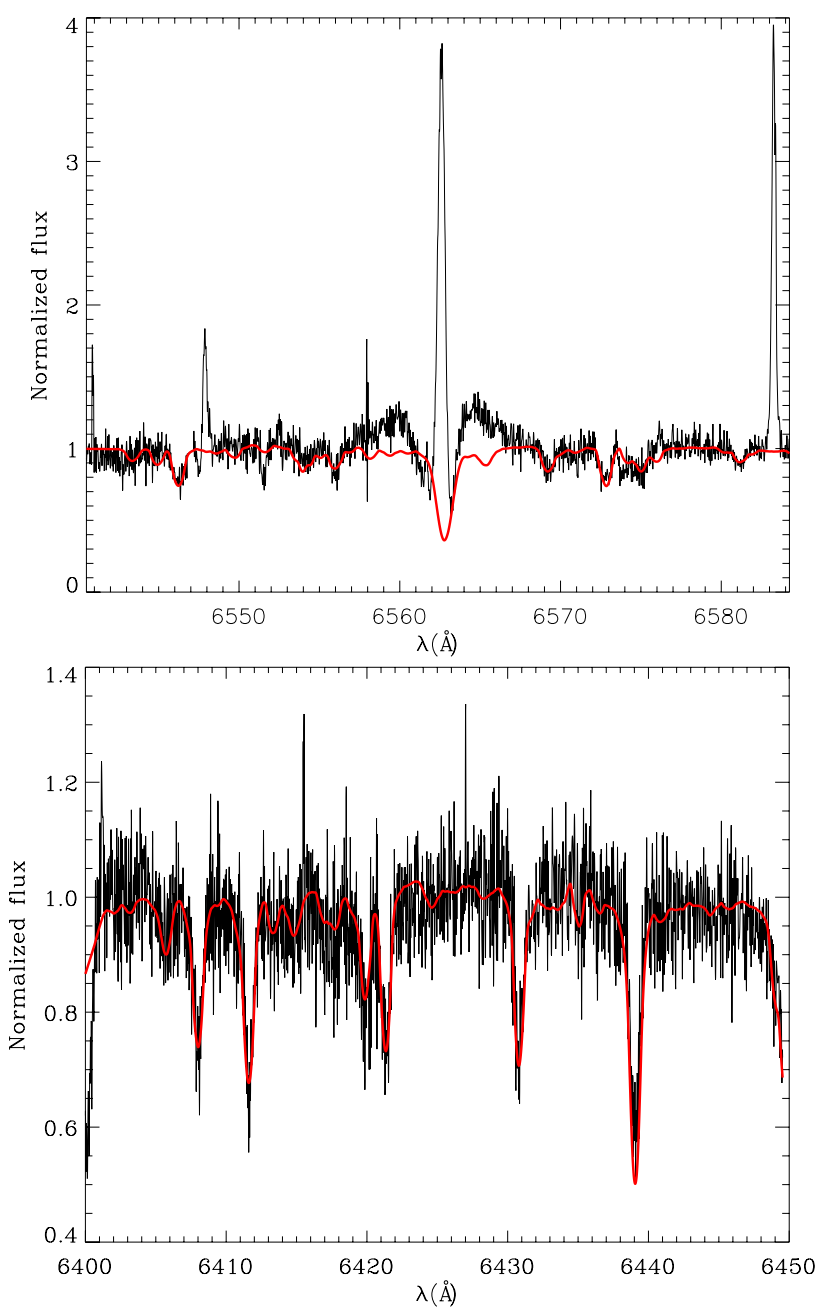

Fig. 7. Two regions of the V354 Mon spectrum (black line) obtained at a photometric maximum on March 12, superimposed on the standard star spectrum (red line), corrected for its radial velocity and rotationally broadened to the V354 Mon value. The photospheric absorptions in the spectrum of both stars agree well, indicating that there is no measurable veiling in the spectrum of V354 Mon. In the top plot, which represents the $\mathrm{H} \alpha$ region, we observe the nebular contribution to this line in the V354 Mon spectrum and the photospheric contribution to $\mathrm{H} \alpha$ in the standard spectrum.

as seen in Fig. 9. According to this result, the hot spot should be facing the observer at light-curve minima, which is not consistent with a hot spot as the main cause of the photometric variation of V354 Mon. Nevertheless, the existence of a hot spot is confirmed by the $\mathrm{H} \alpha$ redshifted absorption, since it indicates that there is material at high velocity that falls onto the star and hits the photosphere, which certainly produces a hot spot at the stellar surface. But this hot spot does not generate a measurable veiling, at least within the error of our measurements, or it may be hidden by the accretion column.

\subsection{Occultation by circumstellar material}

For young stars with an accretion disk seen at high inclination with respect to the line of sight, disk material may absorb part of the stellar flux. If the material is nonuniformly distributed, the light curve is modulated according to the disk structure. Inhomogeneities can be caused by azimuthal asymmetries, a disk with the outer part partially expanded (flared), or 

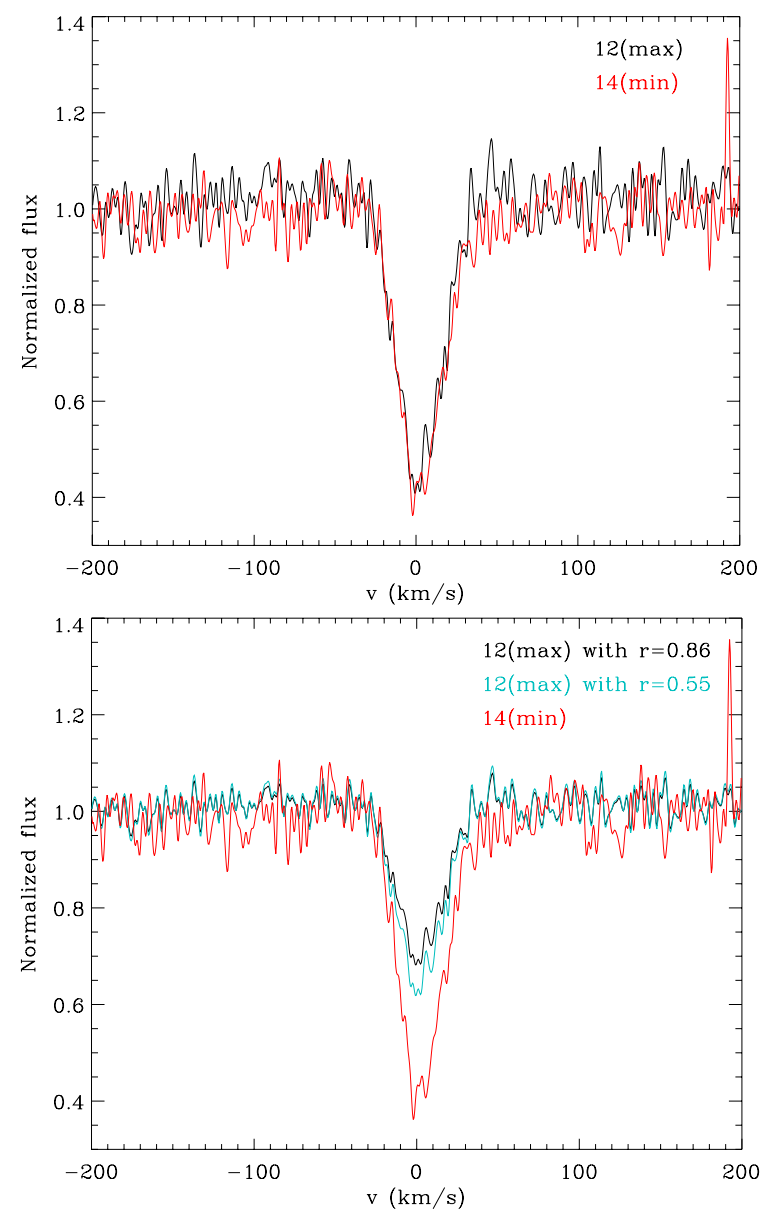

Fig. 8. Comparison between LiI absorption lines observed on March 12 and 14 , corresponding to photometric maximum and minimum. In the top plot there is no difference in line depth, showing that no veiling is detectable with brightness increase. In the bottom plot we added veiling to the spectrum at photometric maximum to demonstrate how a spot with temperature of $10000 \mathrm{~K}$ and $f=5 \%$ would affect the line depth if it were the main cause of the observed photometric variations.

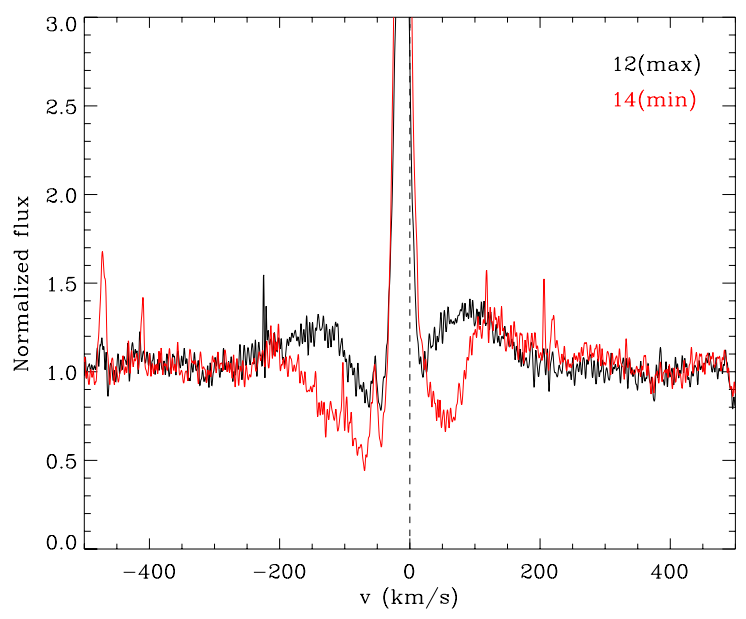

Fig. 9. Comparison between $\mathrm{H} \alpha$ circumstellar spectrum observed on March 12 and 14, corresponding to photometric maximum and minimum. The dashed vertical line indicates the central position of the $\mathrm{H} \alpha$ line. The appearance of a redshifted absorption during the brightness minimum of the star is evident.

the inner part distorted (warped), or even by dust in an inhomogeneous disk wind. In these cases, the timescale of photometric variation depends on the disk angular velocity at the radius where the inhomogeneity is located. Similarly to spots, occultation by circumstellar disk makes the star bluer as it becomes brighter. But there is no color variation with the brightness modulation if the disk material is completely opaque, as observed in AA Tau (Bouvier et al. 1999, 2003, 2007a). The 3D MHD numerical simulations of Romanova et al. (2004) naturally produced deformations in the inner part of the disk when the magnetic axis did not coincide with the rotation axis, creating regions where accretion is favored. This prediction has been confirmed by the study of AA Tau, in which a dynamic interaction between the stellar magnetic field and the inner disk was observed.

The presence of dust around V354 Mon is indicated by observations with the InfraRed Array Camera (IRAC) of Spitzer (Teixeira 2008), used to identify infrared excess emission from hot circumstellar dust. The $\alpha_{\text {IRAC }}$ index represents the slope of spectral energy distribution between $3.6 \mu \mathrm{m}$ and $8 \mu \mathrm{m}$, which is used to classify the inner disk structure, following the criteria proposed by Lada et al. (2006). V354 Mon presents an $\alpha_{\text {IRAC }}=-1.72$, indicating that this star has an optically thick inner disk. The asymmetric shape of minima and maxima observed in the light curve of V354 Mon obtained with CoRoT favors the idea that an irregular structure obscures the light emitted by the star, probably a circumstellar disk with nonuniformly distributed material, where a denser region periodically intercepts the star in our line of sight. This phenomenon can only occur if the disk is seen at high inclination. We calculated the star inclination $i$ through the relation

$\sin i=\frac{P(v \sin i)}{2 \pi R_{*}}$,

where $P$ is the rotation period, $v \sin i$ is the projected rotational velocity, and $R_{*}$ is the stellar radius. Using the effective temperature and luminosity of V354 Mon given by Flaccomio et al. (2006) and the PMS theoretical evolutionary tracks for solar metallicity computed by Siess et al. (2000), we inferred a radius of $2.39 R_{\odot}$, a mass of $1.49 M_{\odot}$, and an age of $2.4 \times 10^{6}$ years. From a cross-correlation analysis of each spectrum with an appropriate numerical mask, we derived a mean rotational velocity of $v \sin i=22.4 \pm 1.2 \mathrm{~km} \mathrm{~s}^{-1}$. Considering the CoRoT photometric period, $P=5.26 \pm 0.50$ days, we obtained $77^{\circ}$ for the inclination $i$ of the stellar rotation axis relative to the line of sight. This means that the system presents a high inclination, which supports the possibility of star occultation by circumstellar material.

The radius, mass, and age of V354 Mon were also estimated from Landin et al. (2006), using a PMS model with non-gray atmosphere, solar metallicity, initially without rotation and applying mixing length theory with $\alpha=2$ to treat the convective energy transport. The result (Table 3) is slightly different from that obtained with the Siess et al. (2000) model, mainly in mass and age, but the values are on the same order of magnitude.

To investigate the possibility of occultation by circumstellar material as the main cause of photometric variability, we applied the model originally developed for AA Tau (Bouvier et al. 1999) to V354 Mon. The obscuring region is identified as a warp, a vertical deformation in the inner part of the circumstellar disk produced by the interaction with the dipole magnetic field inclined with respect to the rotation axis. The occultation model generates a synthetic light curve, assuming that the height of the inner disk varies with the azimuthal position according to

$h(\phi)=h_{\max }\left|\cos \frac{\pi\left(\phi-\phi_{0}\right)}{2 \phi_{\mathrm{c}}}\right|$,

where $\phi_{0}$ is the azimuth of maximum disk height, corresponding to the middle of the eclipse in photometric phase, and $\phi_{\mathrm{c}}$ is the 
Table 3. V354 Mon parameters obtained from two PMS evolutionary models, using the effective temperature and luminosity given by Flaccomio et al. (2006).

\begin{tabular}{lcc}
\hline \hline Model & Siess et al. (2000) & Landin et al. (2006) \\
\hline$R_{*}\left(R_{\odot}\right)$ & 2.39 & 2.35 \\
$M_{*}\left(M_{\odot}\right)$ & 1.49 & 1.12 \\
Age (years) & $2.4 \times 10^{6}$ & $1.2 \times 10^{6}$ \\
$i$ & $77^{\circ}+13^{\circ}$ & $82^{\circ}+8^{\circ}$ \\
$r_{\mathrm{c}}\left(R_{*}\right)$ & $6.1 \pm 1.0$ & $5.6 \pm 1.1$ \\
$v_{\mathrm{ff}}\left(\mathrm{km} \mathrm{s}^{-1}\right)$ & $450 \pm 70$ & $390 \pm 80$ \\
$i_{\mathrm{m}}$ & $74^{\circ} \pm 7^{\circ}$ & $71^{\circ} \pm 10^{\circ}$ \\
\hline
\end{tabular}

Notes. We consider the difference between both models as the error in the radius and mass determination. Therefore, we calculated the errors of $i, r_{\mathrm{c}}, v_{\mathrm{ff}}$, and $i_{\mathrm{m}}$ taking into account the errors in the period, rotational velocity, mass, radius, and $\mathrm{H} \alpha$ redshifted absorption $v_{\max }$.

warp azimuthal semi-extension. Therefore, the height in the inner part of the disk decreases gently from its highest value $h_{\max }$ at $\phi_{0}$ to zero at $\phi_{0} \pm \phi_{\mathrm{c}}$.

Assuming that the photometric period corresponds to the stellar rotation period and the inner disk warp co-rotates with the star, like in AA Tau, the observed photometric period of $5.26 \pm$ 0.50 days would locate the warp at a distance

$r_{\mathrm{c}}=\left(\frac{P}{2 \pi}\right)^{2 / 3}\left(G M_{*}\right)^{1 / 3}=6.1 R_{*}$

from the star, with $M_{*}$ and $R_{*}$ obtained from the model of Siess et al. (2000). At this distance, the magnetic field affects the accretion dynamics and the disk is truncated. Internally to this radius, the flux of material is directed to the star following the field lines. The inclination of the magnetic axis with respect to line of sight can be quantified from the redshifted absorptions in the emission line profiles because they are produced by the accretion funnel material that falls at free-fall velocity in the stellar photosphere. In the spectrum of V354 Mon obtained on March 14, located at the photometric minimum, we observe an $\mathrm{H} \alpha$ redshifted absorption with a maximum velocity, projected on the line of sight, of $125 \pm 25 \mathrm{~km} \mathrm{~s}^{-1}$. The free-fall velocity of the accretion flow material from a truncation radius of $6.1 R_{*}$ is

$v_{\mathrm{ff}}=\left[\frac{2 G M_{*}}{R_{*}}\left(1-\frac{R_{*}}{r_{\mathrm{c}}}\right)\right]^{1 / 2} \simeq 450 \mathrm{~km} \mathrm{~s}^{-1}$,

with $M_{*}$ and $R_{*}$ obtained from the model of Siess et al. (2000). Assuming that the accreting material follows the dipole field lines $^{2}$, the inclination $i_{\mathrm{m}}$ of the magnetic axis is calculated through the relation $\cos i_{\mathrm{m}}=v_{\max } / v_{\mathrm{ff}}$, which gives a value of $\sim 0.28$, i.e., $i_{\mathrm{m}} \simeq 74^{\circ}$. As the inclination of the rotation axis is $\sim 77^{\circ}$, the angle between this and the magnetic axis is only a few degrees at most. The Romanova et al. (2003) MHD simulations have shown that for very small angles of misalignment

\footnotetext{
2 Given the inferred mass and age of V354 Mon, it has already developed a small radiative core. According to Gregory et al. (2012), stars with this configuration present large-scale magnetic fields that are axisymmetric and have high-order components, typically the octupole, that dominate over the dipole at the stellar surface. However, the dipolar component is generally stronger than the octupolar at large distances from the star and then matter flows from the disk towards the star along dipolar lines. The octupole is more important near the stellar surface, redirecting the accretion streams towards the octupolar pole and shaping the hot spots (see the case of V2129 Oph: Donati et al. 2011; Romanova et al. 2011; Alencar et al. 2012).
}
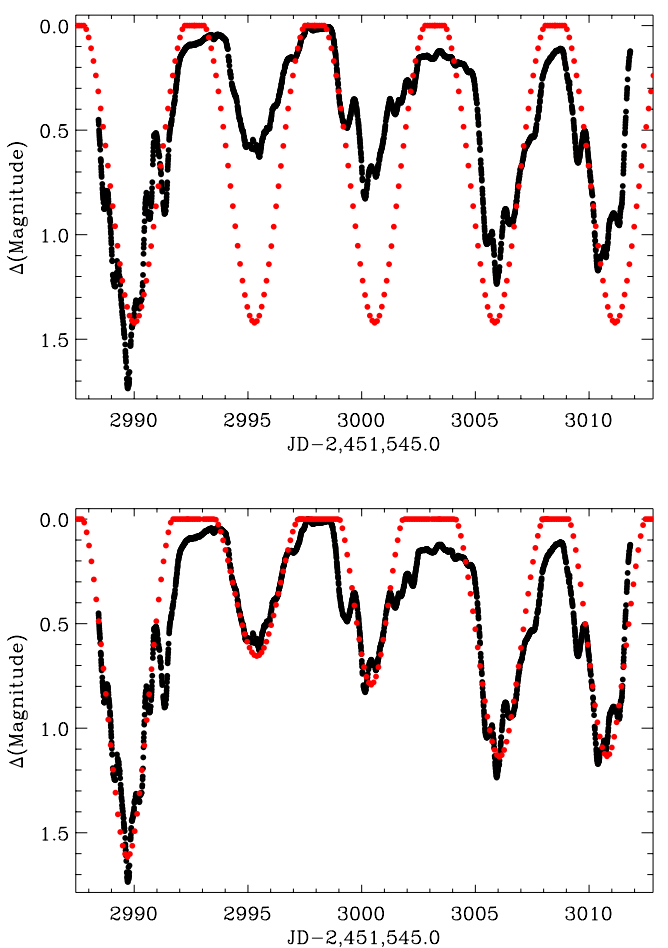

Fig. 10. Best fit of the occultation model with fixed parameters (top), corresponding to a warp with maximum scale height of 0.3 and azimuthal extension of $360^{\circ}$. Individual fit of the model to light-curve minima (bottom). The corresponding properties of the warp are indicated in Table 4.

between the rotation and the magnetic field axes the accretion flows become asymmetric.

We recalculated the inclinations of the rotation and the magnetic field axes using the mass and radius values obtained from Landin et al. (2006) to show the dependence of the results with the adopted theoretical evolutionary model. Even though the radius values given by the two models are very close, the system inclination is affected by the small difference between them. We obtained $i=82^{\circ}$ for $R_{*}=2.35 R_{\odot}$. The inclination of the magnetic field axis with respect to the line of sight is $i_{\mathrm{m}}=71^{\circ}$ and the misalignment with the rotation axis is $11^{\circ}$. We note that the differences between the system parameters obtained from the two theoretical models (Table 3) are negligible, given the expected uncertainties. The results are on the same order of magnitude, confirming that the system is seen at high inclination and the magnetic axis is only slightly tilted with respect to the rotation axis. The misalignment between the magnetic and rotation axes is also observed in other CTTSs (Donati et al. 2010, 2011).

To reproduce the V354 Mon photometric variability observed with CoRoT by employing the occultation model, we considered the mass and radius values derived from Siess et al. (2000). The model free parameters are the warp maximum height $h_{\max }$ and azimuthal semi-extension $\phi_{\mathrm{c}}$, which influence the amplitude of variability and the duration of the eclipse, respectively. With these parameters fixed, the best fit was obtained with $h_{\max }=0.3 r_{\mathrm{c}}$ and a total azimuthal extension of $360^{\circ}$, represented in Fig. 10 (top). The synthetic light curve closely follows the large-scale photometric variation and reproduces the largest amplitudes observed, but does not fit the change in the maxima and minima phase to phase. This is to be expected with fixed warp parameters. However, it is interesting to note that the warp parameters are similar to those obtained in the model of the variability of AA Tau, with a maximum height larger than the value 
Table 4. Occultation model parameters from individual fit of light-curve minima.

\begin{tabular}{lcc}
\hline \hline Minimum & $h_{\max }\left(r_{\mathrm{c}}\right)$ & $2 \phi_{\mathrm{c}}\left({ }^{\circ}\right)$ \\
\hline 1st & 0.31 & 320 \\
2nd & 0.23 & 320 \\
3rd & 0.25 & 240 \\
4th & 0.28 & 320 \\
5th & 0.28 & 280 \\
\hline
\end{tabular}

traditionally used in disk models, $\sim 0.05-0.1 r_{\mathrm{c}}$ (Bertout et al. 1988; Duchêne et al. 2010).

The analysis of AA Tau photometric and spectroscopic variability indicated that the large-scale stellar magnetosphere configuration changed over a month because of the differential rotation between the star and the disk, showing that the circumstellar disk dynamically interacts with the misaligned magnetic field. Therefore, the deformation in the inner disk, which results from this interaction, is also expected to change its characteristics on a short timescale. Based on this and the fact that the depth and width of V354 Mon photometric minima vary considerably, we modeled each light-curve minimum individually. The warp properties are presented in Table 4 for each cycle and the corresponding synthetic light curve is shown in Fig. 10 (bottom). Despite the irregularity of the photometric modulation, the parameters of the inner disk warp for each cycle are quite similar, indicating that small changes in its height and azimuthal extension can reproduce the large amplitude of variability in the observed light curve.

We derived new values of effective temperature and luminosity using the recently published spectral type vs. effective temperature/intrinsic color scale of Pecaut \& Mamajek (2013), which is more appropriate for young stars than the dwarftemperature scales used by Flaccomio et al. (2006). As the $J$-band photometry is less affected by the disk and accretion column emissions than other bands, we used the available 2MASS $J$ magnitude (Skrutskie et al. 2006) and bolometric correction in this band to compute a luminosity of $\log \left(L_{\mathrm{bol}} / L_{\odot}\right)=0.11$. Considering this value and an effective temperature of $4330 \mathrm{~K}$, we recalculated the stellar parameters from the PMS models of Siess et al. (2000) and Landin et al. (2006) (Table 5). Although the mass and radius have decreased compared with the values derived using the effective temperature and luminosity given by Flaccomio et al. (2006) (Table 3), the overall characteristics of the system are on the same order of magnitude. This corroborates the scenario of a young star surrounded by a disk seen at high inclination, with a small misalignement between the magnetic and rotation axes.

The change in the stellar parameters affected the result of the occultation model as the inclination of the system and the corotation radius increased. Considering the mass and radius obtained from Siess et al. (2000), we reproduced exactly the same fit as shown in Fig. 10 (top) with fixed parameters with a warp of maximum scale height of $0.15 r_{\mathrm{c}}$ and total azimuthal extension of $280^{\circ}$. The individual fit of the model to light-curve minima (Fig. 10, bottom) is also recovered with a warp that changes its characteristics from 0.10 to $0.16 r_{\mathrm{c}}$ in maximum height and from $180^{\circ}$ to $260^{\circ}$ in azimuthal extension. As the inclination of the system is slightly steeper in this case, the warp does not need to be too high and too extended to generate the observed amplitude of variations. While the stellar parameters derived using the scale of Pecaut \& Mamajek (2013) are expected to be more suitable for this system, the results in the literature are largely based on the relations compiled by Kenyon \& Hartmann (1995).
Table 5. V354 Mon parameters obtained from two PMS evolutionary models, using the effective temperature and luminosity derived from Pecaut \& Mamajek (2013).

\begin{tabular}{lcc}
\hline \hline Model & Siess et al. (2000) & Landin et al. (2006) \\
\hline$R_{*}\left(R_{\odot}\right)$ & 1.98 & 2.03 \\
$M_{*}\left(M_{\odot}\right)$ & 1.09 & 0.88 \\
Age (years) & $2.5 \times 10^{6}$ & $1.4 \times 10^{6}$ \\
$i$ & $85^{\circ} \pm 5^{\circ}$ & $82^{\circ} \pm 8^{\circ}$ \\
$r_{\mathrm{c}}\left(R_{*}\right)$ & $6.6 \pm 1.0$ & $6.0 \pm 1.0$ \\
$v_{\mathrm{ff}}\left(\mathrm{km} \mathrm{s}^{-1}\right)$ & $420 \pm 50$ & $370 \pm 60$ \\
$i_{\mathrm{m}}$ & $73^{\circ} \pm 7^{\circ}$ & $70^{\circ} \pm 9^{\circ}$ \\
\hline
\end{tabular}

Notes. The process to calculate the errors was the same as described in Table 3.

Therefore we kept the results obtained based on the latter because they are easier to compare with similar published works.

According to the results obtained, occultation by circumstellar material can be the main cause of the photometric variability observed in V354 Mon. Spectroscopic evidence also favors this interpretation. Photons emitted by the accretion shock or lower accretion column are absorbed by the funnel material in free fall, producing a redshifted absorption in $\mathrm{H} \alpha$. As seen from Fig. 9, this absorption is more pronounced in the spectrum that corresponds to a photometric minimum, but it does not appear in the spectrum obtained in the maximum. This is evidence for a correlation between the position of the accretion flow onto the star and the decreasing of stellar brightness. The MHD simulations of Romanova et al. (2003) indicated that for a misalignment between the magnetic field and the rotation axes smaller than $30^{\circ}$, the densest regions of accreting material to the star are located in two main funnels, following the field lines to the closest magnetic pole. Each region is located in one hemisphere, one above the disk and the other below, and rotates with the star. The region located above the disk periodically occults the star for an observer that views the system at medium to high inclination. Therefore, the spectroscopic observations and the CoRoT photometry, along with predictions of numerical simulations, corroborate the idea of stellar occultation by circumstellar material in this system.

A possible explanation for the color variation observed in V354 Mon $B V(R I)_{\mathrm{c}}$ photometry is extinction by circumstellar dust. The star becomes redder when fainter because the warp may not be totally opaque, different from what was observed in AA Tau. An additional analysis is necessary to confirm this hypothesis as the real cause of V354 Mon color variation, which cannot be done based on the data presented in this study.

\section{Conclusions}

From simultaneous high-resolution spectroscopic and photometric observations, we analyzed the CTTS V354 Mon. This star exhibits a large, periodic brightness variation with minima that change in shape from one rotational cycle to the next. A periodogram analysis of the light curve obtained with CoRoT provided a photometric period of $5.26 \pm 0.50$ days, close to the value derived by Lamm et al. (2004), $5.22 \pm 0.87$ days, which indicates that the main structure that produces the photometric modulation did not significantly change over a few years. Observations at $B V(R I)_{\mathrm{c}}$ bands showed that there is also a small color variation and the system becomes slightly bluer as the flux increases.

The spectrum of V354 Mon is variable on a timescale of a few days. The periodicity of $\mathrm{H} \alpha$ redshifted and blueshifted sides supports the magnetospheric accretion scenario, in which the 
star accretes material from the circumstellar disk while it ejects mass through a disk wind that originates close to the accretion region.

We investigated the possibility that spots at the stellar surface are the main source of photometric variations. A spot with a temperature of $10000 \mathrm{~K}$ and occupying $5 \%$ of stellar hemisphere is the spot configuration that best reproduces the variability amplitudes observed in $B V(R I)_{\mathrm{c}}$ bands, which means that the possibility of cold spots is discarded. Such a hot spot would produce a significant veiling in photospheric lines, which is not observed. In addition, the occurrence of pronounced $\mathrm{H} \alpha$ redshifted absorptions seen only in light-curve minima indicates that the accretion funnel and, consequently, the hot spot are visible at this phase, which invalidates this phenomenon as the main cause of photometric modulation.

We found evidence that the emission lines vary in a cyclic manner according to the photometric modulation. The asymmetric shape of light-curve minima and the difference in the spectral line profile at brightness increase and decrease show that an irregular structure produces these variations, probably a circumstellar disk with nonuniformly distributed material. This is supported by the fact that the system is seen at high inclination. According to MHD simulations (Romanova et al. 2004), the small misalignment between magnetic and rotational axes observed in this star may create a distortion in the inner disk, producing a warp that periodically occults part of the stellar photosphere. We used an occultation model to determine the general parameters of this structure, which exhibits a maximum scale height of 0.3 located near the disk corotation point, with an azimuthal extension of $360^{\circ}$. These features are similar to those obtained in the fit of this model to the variability of AA Tau (Bouvier et al. 1999). The warp in the disk of V354 Mon seems to modify its shape at each cycle, revealing a dynamical interaction between the stellar magnetosphere and the disk inner part, as predicted by MHD simulations (Goodson \& Winglee 1999; Romanova et al. 2002). Nevertheless, the parameters obtained from the individual fitting of the model to the light-curve minima are not very different from each other. This result shows that, despite the irregularity of the brightness modulation, small variations in the warp characteristics are capable of reproducing the large amplitude of the observed photometric variability. The presence of an $\mathrm{H} \alpha$ redshifted absorption component more pronounced only at photometric minimum points to a connection between the accretion funnel and the flux variations of the system, which corroborates that occultation by circumstellar material is the main cause of photometric modulation in V354 Mon.

Acknowledgements. We thank Suzanne Aigrain for pre-processing the CoRoT light curve and the anonymous referee for many useful comments and suggestions. N.N.J.F. acknowledges support from CAPES (fellowship process No. 18697-12-7) and CNPq. S.H.P.A. acknowledges support from CAPES, CNPq, and Fapemig. J.B. acknowledges funding from CNES and from ANR Toupie 2011 Blanc SIMI5-6 020 01. This research was developed within the scope of CAPES-Cofecub project and is based on data collected in the CoRoT satellite.

\section{References}

Alencar, S. H. P., Teixeira, P. S., Guimarães, M. M., et al. 2010, A\&A, 519, A88 Alencar, S. H. P., Bouvier, J., Walter, F. M., et al. 2012, A\&A, 541, A116
Auvergne, M., Bodin, P., Boisnard, L., et al. 2009, A\&A, 506, 411

Bertout, C., Basri, G., \& Bouvier, J. 1988, ApJ, 330, 350

Bessell, M. S. 1983, PASP, 95, 480

Bouvier, J., Cabrit, S., Fernandez, M., Martin, E. L., \& Matthews, J. M. 1993, A\&A, 272, 176

Bouvier, J., Covino, E., Kovo, O., et al. 1995, A\&A, 299, 89

Bouvier, J., Chelli, A., Allain, S., et al. 1999, A\&A, 349, 619

Bouvier, J., Grankin, K. N., Alencar, S. H. P., et al. 2003, A\&A, 409, 169

Bouvier, J., Alencar, S. H. P., Boutelier, T., et al. 2007a, A\&A, 463, 1017

Bouvier, J., Alencar, S. H. P., Harries, T. J., Johns-Krull, C. M., \& Romanova, M. M. 2007b, Protostars and Planets V, 479

Cabrit, S., Edwards, S., Strom, S. E., \& Strom, K. M. 1990, ApJ, 354, 687

Claret, A. 2000, A\&A, 363, 1081

Cody, A. M., Stauffer, J., Baglin, A., et al. 2014, AJ, 147, 82

Dahm, S. E. 2008, The Young Cluster and Star Forming Region NGC 2264, ed. B. Reipurth, 966

Dahm, S. E., \& Simon, T. 2005, AJ, 129, 829

Donati, J.-F., Skelly, M. B., Bouvier, J., et al. 2010, MNRAS, 409, 1347

Donati, J.-F., Bouvier, J., Walter, F. M., et al. 2011, MNRAS, 412, 2454

Duchêne, G., McCabe, C., Pinte, C., et al. 2010, ApJ, 712, 112

Fürész, G., Hartmann, L. W., Szentgyorgyi, A. H., et al. 2006, ApJ, 648, 1090

Flaccomio, E., Micela, G., \& Sciortino, S. 2006, A\&A, 455, 903

Gillen, E., Aigrain, S., McQuillan, A., et al. 2014, A\&A, 562, A50

Goodson, A. P., \& Winglee, R. M. 1999, ApJ, 524, 159

Gregory, S. G., Donati, J.-F., Morin, J., et al. 2012, ApJ, 755, 97

Hartigan, P., Edwards, S., \& Ghandour, L. 1995, ApJ, 452, 736

Hartmann, L., Hewett, R., \& Calvet, N. 1994, ApJ, 426, 669

Henney, W. J., \& O'Dell, C. R. 1999, AJ, 118, 2350

Herbig, G. H. 1954, ApJ, 119, 483

Herbst, W., Herbst, D. K., Grossman, E. J., \& Weinstein, D. 1994, AJ, 108, 1906

Johns, C. M., \& Basri, G. 1995, AJ, 109, 2800

Johns-Krull, C. M., Valenti, J. A., Piskunov, N. E., Saar, S. H., \& Hatzes, A. P. 2001, ASPC, 248, 527

Kenyon, S. J., \& Hartmann, L. 1995, ApJS, 101, 117

Kurosawa, R., Harries, T. J., \& Symington, N. H. 2006, MNRAS, 370, 580

Lada, C. J., Muench, A. A., Luhman, K. L., et al. 2006, AJ, 131, 1574

Lamm, M. H., Bailer-Jones, C. A. L., Mundt, R., Herbst, W., \& Scholz, A. 2004, A\&A, 417, 557

Landin, N. R., Ventura, P., D’Antona, F., Mendes, L. T. S., \& Vaz, L. P. R. 2006, A\&A, 456, 269

Lima, G. H. R. A., Alencar, S. H. P., Calvet, N., Hartmann, L., \& Muzerolle, J. 2010, A\&A, 522, A104

Mariñas, N., Lada, E. A., Teixeira, P. S., \& Lada, C. J. 2013, ApJ, 772, 81

McCollum, B., Bruhweiler, F., Castelaz, M. W., et al. 2004, BAAS, 36, 1519

Ménard, F., Bouvier, J., Dougados, C., Mel'nikov, S. Y., \& Grankin, K. N. 2003 A\&A, 409, 163

Muzerolle, J., Calvet, N., \& Hartmann, L. 2001, ApJ, 550, 944

Pecaut, M. J., \& Mamajek, E. E. 2013, ApJS, 208, 9

Perruchot, S., Kohler, D., Bouchy, F., et al. 2008, in SPIE Conf. Ser., 7014

Romanova, M. M., Ustyugova, G. V., Koldoba, A. V., \& Lovelace, R. V. E. 2002, ApJ, 578, 420

Romanova, M. M., Ustyugova, G. V., Koldoba, A. V., Wick, J. V., \& Lovelace, R. V. E. 2003, ApJ, 595, 1009

Romanova, M. M., Ustyugova, G. V., Koldoba, A. V., \& Lovelace, R. V. E. 2004, ApJ, 610, 920

Romanova, M. M., Long, M., Lamb, F. K., Kulkarni, A. K., \& Donati, J.-F. 2011 , MNRAS, 411, 915

Samadi, R., Fialho, F., Costa, J. E. S., et al. 2007 [arXiv: 0703354]

Scargle, J. D. 1982, ApJ, 263, 835

Shu, F., Najita, J., Ostriker, E., et al. 1994, ApJ, 429, 781

Siess, L., Dufour, E., \& Forestini, M. 2000, A\&A, 358, 593

Skrutskie, M. F., Cutri, R. M., Stiening, R., et al. 2006, AJ, 131, 1163

Sung, H., Bessell, M. S., \& Lee, S. 1997, AJ, 114, 2644

Teixeira, P. S. 2008, Ph.D. Thesis, Harvard-Smithsonian Center for Astrophysics, USA and University of Lisbon, Portugal

Terquem, C., \& Papaloizou, J. C. B. 2000, A\&A, 360, 1031

Valenti, J. A., \& Piskunov, N. 1996, A\&AS, 118, 595

Walker, M. F. 1956, ApJS, 2, 365 